\title{
THE FUNCTIONAL ROLE OF CONTRAST ADAPTATION*
}

\author{
Mark W. Greenlee and Friedrich Heitger \\ Neurologische Universitätsklinik mit Abteilung für Neurophysiologie, Hansastr. 9, 7800 Freiburg im Br., \\ F.R.G.
}

(Received 17 August 1987; in revised form 17 Decemher 1987)

\begin{abstract}
Prolonged inspection of high contrast sinewave gratings increases the contrast required to detect gratings having a similar spatial frequency and orientation. The functional role of such adaptation has, however, in the past, eluded disclosure. We here show that $5 \mathrm{~min}$ adaptation to a $2 \mathrm{c} / \mathrm{deg}$ sinewave grating of 0.8 contrast changes the observer's ability to discriminate the contrast level of a subsequently presented grating of the same spatial frequency and orientation. Similar to the threshold elevation effect, the observers required more incremental contrast for background contrast levels between 0.1 and 0.4 following adaptation. However, for contrast levels above 0.5 , the observers required less delta contrast, following adaptation, to correctly discriminate which of two gratings was incremented in contrast. A simple model for adaptation is proposed to account for the findings which is based on a shift in the semi-saturation constant of the detector's contrast-response function. According to this model, adaptation acts to linearize the underlying mechanism's response in the region near the prevailing contrast level.
\end{abstract}

\section{INTRODUCTION}

Prolonged inspection of high contrast gratings increases the contrast threshold for detecting gratings of similar spatial frequency and orientation (Pantle and Sekuler, 1968; Blakemore and Campbell, 1969) and reduces the perceived contrast of subsequently presented gratings (Blakemore et al., 1973; Georgeson, 1985). Such consequences of adaptation appear to have a detrimental effect on perception by decreasing or biasing postadaptation sensitivity.

Earlier attempts to explore the possible benefits of contrast adaptation failed to disclose an enhanced sensitivity to differences in contrast near the adapting contrast (Barlow et al., 1976; Kulikowski and Gorea, 1978; Legge, 1981). Barlow et al. (1976) adapted their observers to a $6 \mathrm{c} / \mathrm{deg}$ sinewave grating of 0.5 contrast and tested their ability to discriminate briefly presented gratings differing slightly in contrast. One observer showed no effect of adaptation and the second observer needed slightly more incremental contrast to be able to discriminate the gratings. Kulikowski and Gorea (1978) found that adaptation was required if Weber's law should hold for contrast discrimination, implying that adaptation increases discrimi-

\footnotetext{
*Part of these findings were presented at the 9th European Vision Conference, September 1986, in Bad Nauheim, F.R.G.
}

nation thresholds. Legge (1981), using several background contrast levels, with and without adaptation, could not find any difference caused by adaptation.

Interestingly, single-unit recordings in cat cortex before and after contrast adaptation indicate the shift in the contrast response function expected if adaptation should have an advantageous effect on contrast discrimination thresholds (Albrecht and Hamilton, 1983; Dean, 1983; Albrecht et al., 1984; Ohzawa et al., 1985; Sclar et al., 1985). Ohzawa et al. (1985) suggest that adaptation acts to adjust the contrast gain of the underlying neural mechanisms. Psychophysical findings by Georgeson and Georgeson (1987) further show that shifts in sensitivity occur even after very brief exposures to spatial contrast, although the duration of such aftereffects is very short compared to that induced by prolonged adaptation (Magnussen and Greenlee, 1985). These latter findings suggest that the adaptation effects measured psychophysically may reflect the dynamic nature of the underlying detector's contrast response function.

We here report findings of experiments that compared the observer's ability to discriminate between two gratings differing slightly in contrast for various background contrast levels before and after adaptation to a high-contrast $(0.8)$ grating. The findings show that adaptation increases the contrast discrimination threshold for background contrast levels below 0.5 and decreases discrimination contrast thresholds for 
background contrast levels of 0.5 and above. A simple model based on a shift in the contrast response function resulting in a linear response to all contrast levels is suggested to account for our results.

\section{METHOD}

Sinusoidal luminance gratings were generated on a high-resolution cathode ray tube (Joyce Electronics, Cambridge, U.K.) having a white (P4) phosphor with a space-averaged luminance of $100 \mathrm{~cd} / \mathrm{m}^{2}$. Analog signals under microprocessor control determined the spatial frequency, spatial phase and contrast of the gratings. Grating contrast was defined as the difference between the maximal and minimal luminance levels divided by their sum. A photometer was used before each session to assure that the display had a linear contrast characteristic up to the highest contrast level presented (0.9). The display was viewed binocularly with natural pupils at a constant viewing distance of $114 \mathrm{~cm}$, at which distance it subtended 11 by $15 \mathrm{deg}$ at the eye. The display was surrounded by a uniformly illuminated semi-circular screen of $50 \mathrm{~cd} / \mathrm{m}^{2}$ luminance level. The observer rested his chin and forehead on a support to assure constant viewing distance and head orientation.

Before and after adaptation contrast discrimination thresholds were measured using a temporal two alternative forced-choice procedure. Test gratings were presented for $1 \mathrm{sec}$ in two intervals, delineated by auditory signals and separated by $1 \mathrm{sec}$ with a uniform field of zero contrast. Before adaptation test trials were interleaved with $15 \mathrm{sec}$ exposures to a uniform field, whereas after adaptation test trials were followed by $15 \mathrm{sec}$ exposure to the adapting grating. The latter has proved to maintain the postadaptation level at a steady state (Magnussen and Greenlee, 1986). Observers adapted for $5 \mathrm{~min}$ to a high-contrast $(0.8)$ sinewave grating of vertical orientation and a spatial frequency of $2.0 \mathrm{c} / \mathrm{deg}$. To cancel afterimages and to create an adaptation effect which was independent of the spatial phase of the test gratings, we adapted our observers to slowly $(0.5 \mathrm{~Hz})$ drifting adapting gratings, the drift direction of which changed every $4 \mathrm{sec}$. The test gratings were also drifted at this rate, the initial direction of which was randomly chosen by the computer. The contrast of the test gratings was fixed at a defined background level and the computer selected on a random basis in which interval an increment in contrast was added. The amplitude of this increment was determined in a staircase procedure using a maximumlikelihood algorithm (Lieberman and Pentland. 1982).

In the first set of experiments, the contrast of one of the two test gratings was incremented $0.5 \mathrm{sec}$ after the onset of the test grating. Here the subjects were asked to detect which of the gratings was incremented in contrast during its exposure. Figure la (upper panel) shows the temporal characteristic of this procedure. In a second experiment, the background contrast and the added contrast increment were presented simultaneously. Here the observer had to discriminate which of the two gratings had the larger contrast. This condition is illustrated in Fig. Ib (lower panel). In the first experiment the sudden increment in contrast was therefore used as a criterion for discrimination, whereas in the second experiment the subjects had from memory to compare the contrast of the first and second grating. All other parameters were otherwise identical throughout the experiments.

\section{RESULTS}

\section{Contrast discrimination before and after adaptation}

First we measured the subject's ability to detect a slight increment in the contrast of a grating as a function of the background contrast

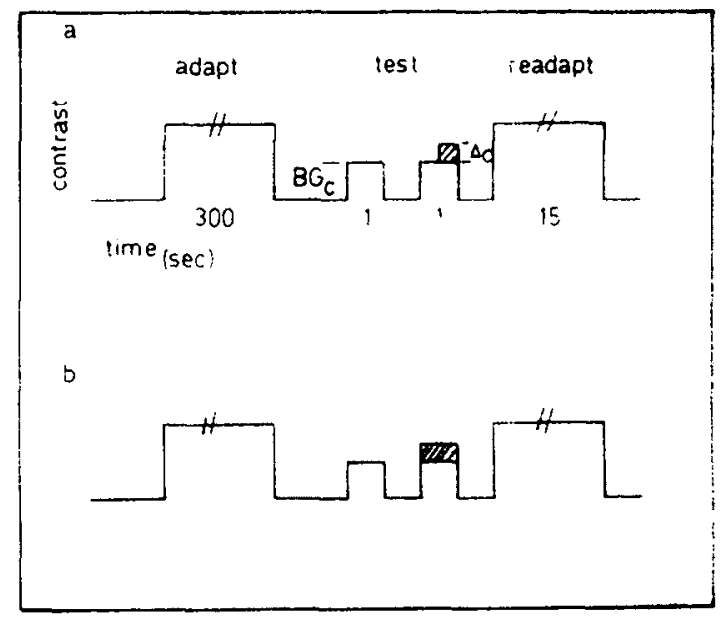

Fig. I. Schematic illustration of the contrast/time profiles used in the two experiments. Upper panel (a) depicts the condition where test contrast is incremented during presentation of the test grating and the lower panel (b) shows the condition where the one of the test gratings is presented with more contrast. 

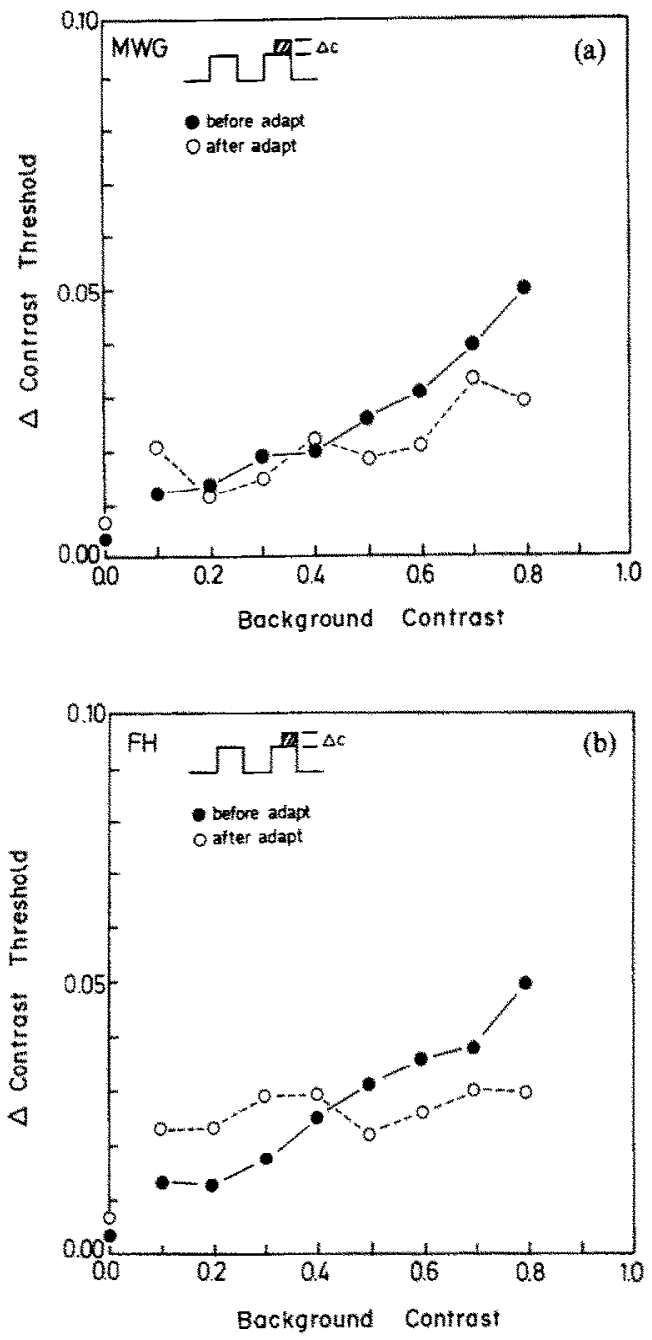

Fig. 2. Discrimination contrast thresholds are plotted on linear axes as a function of the background contrast of vertically oriented sinewave gratings of $2 \mathrm{c} / \mathrm{deg}$ spatial frequency. Filled circles show the values measured before adaptation and the open circles show discrimination thresholds measured on the test-readapt schedule following $5 \mathrm{~min}$ adaptation to a 0.8 contrast grating of the same orientation and spatial frequency. The inset shows the temporal characteristic of the test gratings. Values for observer MWG are given in (a) and those for observer FH in (b).

level of that grating. The results are shown in Fig. $2 a$ for observer MWG as filled circles. The amount of incremental contrast required to discriminate the test gratings is plotted as a function of the background contrast of these gratings on linear axes. The contrast thresholds, that is the amount of contrast required to discriminate a grating from a blank field, are shown by the data points on the ordinate. The data shown represent the mean of two separate runs and the variability of the threshold esti- mates usually did not exceed $0.05 \log$ unit or $10 \%$ of the background contrast. The lowest background contrast level used was 0.1 so the well-established "dipper" function (Kulikowski and Gorea, 1978) has not been considered here. The findings indicate that with increasing background levels more contrast is required to detect an increment. A 4-5-fold increase in the discrimination threshold was found for the two subjects tested for background contrast levels varying from 0.1 to 0.8 . This is in good agreement with earlier findings (Legge, 1981). Results from a second subject (FH) are shown in Fig. $2 b$ and are in similar to those from MWG.

The open circles in Fig. 2 present the results obtained after $5 \mathrm{~min}$ adaptation to a slowly drifting adapting grating of 0.8 contrast. The open circle on the ordinate gives the detection threshold following adaptation and the difference between thresholds before and after adaptation yields the contrast threshold elevation. This corresponds to a $0.4 \log$ unit elevation, on average, for the two subjects tested, which is in good agrcement with earlier studies (Blakemore and Campbell, 1969; Magnussen and Greenlee, 1985). For background contrast levels between 0.1 and 0.4 , discrimination thresholds are only slightly, or not at all, elevated by adaptation for subject MWG, whereas FH shows a more pronounced elevation in the discrimination threshold for background contrast levels between 0.1 and 0.4 . Adaptation to the highcontrast grating decreased the contrast discrimination threshold for both subjects for contrast levels between 0.5 and 0.8 . The greatest improvement in the discrimination threshold was found for the highest background contrast level $(0.8)$, where the threshold for detecting an increment in contrast is reduced to about half of the unadapted value.

Figure 3 shows, for observer MWG, the results of the second experiment, where delta contrast was presented simultaneously with the background contrast. As in Fig. 2, the filled circles present discrimination thresholds measured prior to adaptation and the open symbols shows the results obtained after $5 \mathrm{~min}$ adaptation on the test-readapt schedule. Compared to the findings shown in Fig. 2, the overall thresholds are higher and the slope of the discrimination contrast threshold vs background contrast function is steeper for the measurements obtained before adaptation. The effect of adaptation in this experiment is even more pronounced than for the experiment 


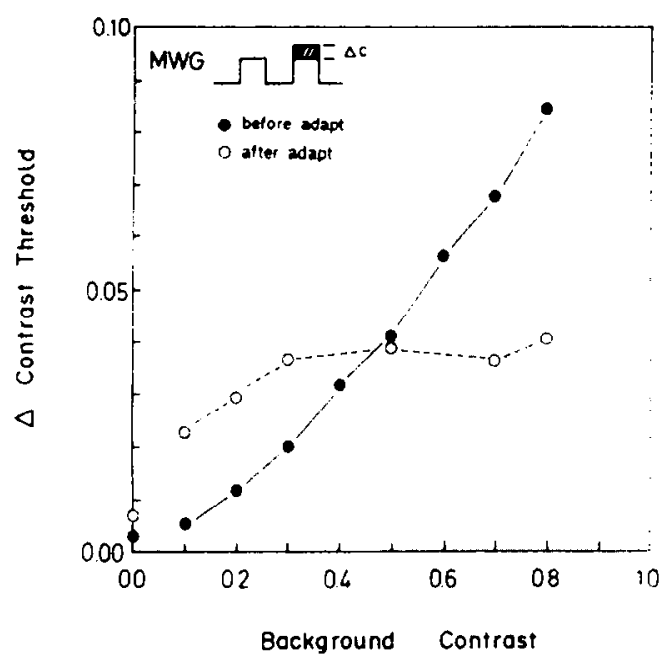

Fig. 3. Contrast discrimination thresholds for subject MWG are shown for the experiment using a different temporal onset of the contrast increment (see inset). As in Fig. 2, filled circles present values measured before adaptation and open circles give the values measured on the test-readapt schedule after $5 \mathrm{~min}$ adaptation.

where contrast was incremented during the presentation of the test grating. Discrimination threshold for the 0.8 background contrast is about a factor of two lower following adaptation.

Orientational selectivity of adaptation effect on discrimination thresholds

The elevation in contrast threshold is characterized by stimulus selectivity along the dimensions of spatial frequency and orientation (Gilinski, 1968; Blakemore and Campbell. 1969), and transfers to the unadapted eye (Gilinsky and Doherty, 1969; Björklund and Magnussen, 1981), suggesting a cortical origin of the effect. We were interested to explore whether the enhancement in contrast discrimination found here for background contrast levels between 0.5 and 0.8 displayed a selectivity to the orientation of the adapting and test gratings. Such orientational selectivity has been repeatedly found in the responses of cortical cells in the cat (Campbell et al., 1968) and the monkey (De Valois et al., 1982). To explore this aspect, we adapted our observers to a vertically oriented grating of 0.8 contrast. Before and after this adaptation contrast discrimination thresholds were measured for vertically and horizontally oriented gratings having the same spatial frequency. The thresholds were measured before and after adaptation as described in the method section (Fig. 1a), with the exception that after adaptation to a vertical grating the test grating was presented either in the same or in the orthogonal orientation. This was done by first blanking the screen and then rotating the deflection coil by 90 angular degrees via a servo motor under computer control. After the test presentation the deflection coil was returned to its original position and the readaptation period was initiated. Care was taken to assure that only the vertical and horizontal orientations were presented.

The results of this experiment are shown in Fig. 4 for both subjects tested (data for MWG, open columns, for $\mathbf{F H}$, hatched columns). Mean threshold values \pm 2 SE of the mean are shown. A single background contrast level of 0.8 was used and the spatial frequency of the adapting and test gratings was $2.0 \mathrm{c} / \mathrm{deg}$. The contrast discrimination thresholds are shown for measurements taken before adaptation (left), after 5 min adaptation to a vertical grating for vertical test gratings (middle), and after $5 \mathrm{~min}$ adaptation to a vertical adapting gratings for horizontal test gratings (right). There is a slight effect on the orthogonally oriented test gratings, but this effect is much less than that found for test gratings of the same (vertical) orientation. A slight effect at orthogonal orientations has also been reported for the elevation in contrast threshold (usually around $0.1 \mathrm{log}$ unit; see Blakemore and Campbell, 1969: Greenlee $c^{\prime} t$ al. 1988).

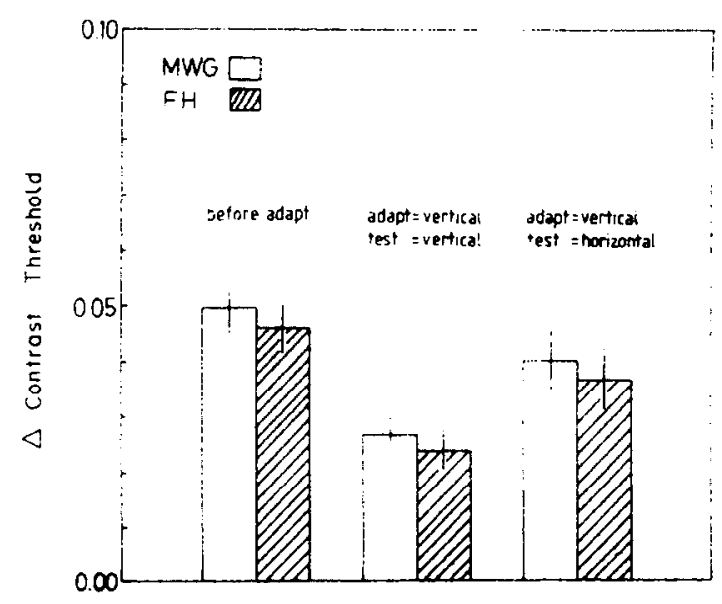

Fig. 4. Contrast discrimination thresholds measured for a $2.0 \mathrm{c} / \mathrm{deg}$ sinewave grating of 0.8 contrast under the following conditions: before adaptation (left), after 5 min adaptation to a vertical grating using vertically oriented test gratings (middle), and after 5 min adaptation to a vertical grating using horizontally oriented test gratings (right). For thexemporal characteristic of the test gratings and the contrast increment see Fig. 1a. Mean thresholds are shown and error bars represent $\pm 2 S E$. 


\section{DISCUSSION}

The present findings indicate that adaptation can enhance an observer's ability to detect an increment in contrast or to discriminate the contrast of two gratings presented in sequence. The enhancement found in discrimination thresholds only holds for background contrast levels of 0.5 and higher. Below 0.5 background contrast, if anything, an increase in the increment contrast thresholds was found. In light of these results, it is obvious why Barlow et al. (1976) failed to find an effect of adaptation on contrast discrimination. The adaptation level they explored $(0.5)$ is directly in the region where we found the pre- and postadaptation curves to cross. Thus, although Barlow et al. (1976) were correct in concluding that, under their conditions, adaptation had no beneficial effects on contrast discrimination thresholds, this conclusion is only true for the background contrast level of about 0.5 .

Legge (1981) used several background levels with and without adaptation and also concluded that adaptation had no faciliatory effect on discrimination thresholds. However, he only measured up to a 0.24 contrast background level, the 0.48 measurement lacking for both subjects after adaptation in his Fig. 2 (spatial frequency, $2 \mathrm{c} / \mathrm{deg}$ ). The $\log -\log$ plot used by Legge also tends to visually underestimate the differences caused by adaptation for higher contrast levels, but following adaptation the discrimination thresholds are first elevated for low background contrasts and then tend to cross the preadapted values for higher levels. In agreement with Legge's findings, we found a slight increase in discrimination thresholds after adaptation for values below 0.5 contrast.

The single-unit findings of Ohzawa et al. (1985) and Sclar et al. (1985) are of interest here as these authors explicitly examined the effects of adaptation in light of the gain adjustment hypothesis. They found that adaptation enhanced the response of cortical, but not LGN, cells to changes in the prevailing contrast level. The present findings would suggest that this enhanced sensitivity to changes in contrast in cells in the visual cortex may play a role in the enhanced ability to detect incremental contrast or to discriminate gratings presented sequentially with slightly different contrast levels. The ability of cortical neurons to adjust their gain dependent on their prior activity would be advantageous in a system encoding relative changes in contrast, but would make the output less reliable with respect to absolute contrast. The findings of Ohzawa et al. (1985) and others suggest that the response of cortical neurons saturates at high contrast levels and that the semi-saturation constant is related to the contrast threshold of the unit under investigation: sensitive cells saturate at lower contrast levels than do cells with high contrast thresholds. The adaptability of the cell's response allows for greater stimulus differentiation along the adapted parameter, here contrast, in the region where response compression would occur, if no gain adjustment took place.

There are, however, important differences between the present psychophysical results and the single-unit findings by Ohzawa et al. (1985). First, Ohzawa et al. (1985) found a shift in the contrast response function after adaptation to contrasts as low as 0.03 , whereas, using a high $(0.8)$ adapting contrast, we found the greatest effects at contrast levels above 0.5. Second, although they found the majority of simple and complex cells to be adaptable, several cortical cells in Ohzawa et al.'s sample showed low adaptability, which would presumably affect psychophysical measurements. More recent work (Sclar and Lennie, 1987) even suggests differences between cat and monkey cortex. Therefore, although there are some similarities between the single-unit recording in cat cortex and psychophysical recording in humans, the differences suggest that caution should be taken when making such comparisons.

\section{The functional role of contrast adaptation}

Our findings and earlier results (Barlow et al., 1976; Kulikowski and Gorea, 1978; Legge, 1981) clearly show that the contrast response function of the human visual system shows a saturating characteristic. A perfectly linear contrast response function would result in a flat function in Fig. 2, i.e. discrimination thresholds would be constant. Such linearity is obtained in observer FH, and nearly so for observer MWG, following adaptation to a high-contrast grating of the same spatial frequency and orientation. Thus, although sensitivity decreases after adaptation for low contrast levels, it increases for the contrast levels where the greatest response compression occurs in the unadapted state, namely at 0.5 contrast and above.

Figure 5 presents a simple descriptive model to account for our results. Here the response amplitude of the underlying mechanism is 
shown (on linear axes) to covary with variations in the stimulus contrast in a nonlinear compressive fashion $\left(\mathrm{crf}_{0}\right)$. The contrast "threshold" of the detector is the response amplitude that significantly exceeds the spontaneous noise level of the mechanism (noise level denoted by the horizontal arrow in Fig. 5). Above threshold, a significant increment in the response level would convey a corresponding increase in the contrast level of the stimulus. The steeper the slope of the contrast response function, the less contrast will be required for the detector to signal a change in stimulus contrast $\left(\Delta c_{1}\right)$. Since the contrast response is normally compressive for contrast levels above 0.5 , more contrast is required in order for the mechanism to detect a change in the prevailing contrast level $\left(\Delta c_{2}\right)$. Adaptation to a high-contrast stimulus is depicted as a shift in the response function to the right towards higher contrast levels $\left(\mathrm{crf}_{\mathrm{a}}\right)$. This shift acts to raise the contrast threshold $\left(\mathrm{CT}^{\prime}\right)$, but at the same time increases the slope in the upper contrast region where the mechanism was formerly saturated $\left(\Delta c_{2}^{\prime}\right)$. Thus, the more adaptable the system is, the more dynamic the response function is, and the more linear becomes the resultant contrast discrimination performance of the mechanism, although some

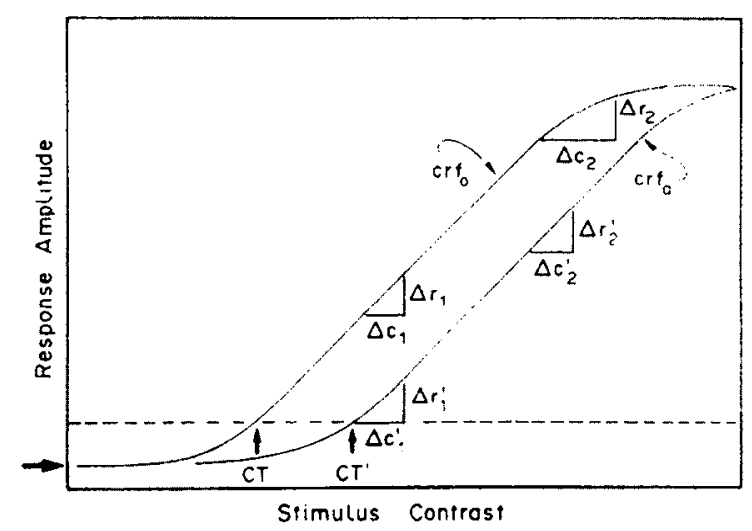

Fig. 5. Hypothetical contrast response functions are depicted before $\left(\mathrm{crf}_{0}\right)$ and after $\left(\mathrm{crf}_{\mathrm{a}}\right)$ adaptation of the supposed underlying neural mechanisms. The contrast "threshold" of this mechanism is denoted by the dashed horizontal line, which is significantly higher than the spontaneous activity of the mechanism (horizontal arrow). Adaptation to a 0.8 contrast grating, whose spatial frequency and orientation fall within the bandwidth of the mechanism studied here, is shown to shift the response function towards higher contrast levels. The amount of incremental contrast required to elicit a significant change in the response amplitude of the detector is shown to increase for low contrast levels $\left(\Delta c_{l}^{\prime}\right)$ and to decrease for high contrast levels $\left(\Delta c_{2}^{\prime}\right)$ following adaptation. information is lost about absolute contrast levels (Blakemore et al., 1973). A similar model has been recently applied to account for changes in VEP-amplitude following contrast adaptation (Bach et al., 1988).

In summary we conclude that contrast adap. tation not only increases the contrast threshold for gratings having similar spatial characteristics, but also improves the contrast discrimination function in the region where the system would otherwise show the greatest response compression. This enhancement is selective to the orientation of the test grating implying that the mechanism involved is probably cortical in origin. A dynamic, stimulus-dependent contrast response function would be useful in a nonlinear system which should be optimally sensitive to near-threshold contrast levels, but should also be able to discriminate between two variously high contrast levels.

Acknowledgements---This research was supported by the Deutsche Forschungsgemeinschaft (SFB 325, B4). The authors thank L. Spillmann for providing laboratory facilities and heipful comments.

\section{REFERENCES}

Albrecht D. B. and Hamilton D. B. (1983) Striate cortex of monkey and cat: contrast response functions. $J$. Neurophysiol. 48, 217-237.

Albrecht D. B., Farrar S. B. and Hamilton D. B. (1984) Spatial contrast adaptation characteristics of neurones recorded in the cat's visual cortex. J. Physiol., Lond. 347, $713 \cdots 737$.

Bach M., Greenlee M. W. and Bühler B. (1988) Contrast adaptation can increase visually evoked potential amplitude. Clin. Vision Sci. (In press).

Barlow H. B., MacLeod D. I. A. and van Meeteren A. (1976) Adaptation to gratings: no compensatory advantages found. Vision Res. 16, 1043-1045

Björklund R. A and Magnussen S. (1981) A study of the interocular transfer of adaptation. Perception 10. $511-518$

Blakemore C. and Campbell F. W. (1969) On the existence of neurones in the human visual system selectively sensitive to the orientation and size of retinal images. J. Physiol, Lond. 203, 237-260

Blakemore C., Muncey J. P. J. and Ridley R. M. (1973) Stimulus specificity in the human visual system. Vision Res. 13, 1915-1931.

Campbell F. W.. Cleland B. G.. Cooper G. F. and EnrothCugell $C$. (1968) The angular selectivity of visual cortical cells to moving gratings. J. Physiol., Lond. 198, 237-250.

Dean D. F. (1983) Adaptation-induced alteration of the relation between response amplitude and contrast in cat striate cortical mechanisms. Vision Res. 23, 249-256

De Valois R. L., Yund $W$. and Hepler N. (1982) The orientation and directional selectivity of cells in the macaque visual cortex. Vision Res. 22, 531-544.

Georgeson M. A. (1985) The effect of spatial adaptation on perceived contrast. Spatial Vision 1, 103-112 
Georgeson M. A. and Georgeson J. M. (1987) Facilitation and masking of briefly presented gratings: time course and contrast dependence. Vision Res. 27, 369-379.

Gilinsky A. S. (1968) Orientation-specific effects of patterns of adapting lights on visual acuity. $J$. opt. Soc. Am. 58, 13-18.

Gilinsky A. S. and Doherty R. S. (1969) Interocular transfer of orientational effects. Science, N.Y. 164, 454-455.

Greenlee M. W., Magnussen S. and Nordby K. (1988) Spatial vision of the achromat: spatial-frequency and orientation specific adaptation. J. Physiol, Lond. 395 661678.

Kulikowski J. J. and Gorea A. (1978) Complete adaptation to patterned stimuli: a necessary and sufficient condition for Weber's law for contrast. Vision Res. 18 , $1223-1227$.

Legge G. E. (1981) A power law for contrast discrimination. Vision Res. 21, 457-467.

Lieberman H. R. and Pentland A. P. (1982) Micro- computer-based estimation of psychophysical thresholds: the Best PEST. Behav. Res. Meth. Instrum. 14, 21-25.

Magnussen S. and Greenlee M. W. (1985) Marathon adaptation to spatial contrast: saturation in sight. Vision Res. 25, 1409-1411.

Magnussen S. and Greenlee M. W. (1986) Contrast threshold elevation following continuous and interrupted adaptation. Vision Res. 26, 673-675.

Ohazawa I., Sclar G. and Freeman R. D. (1985) Contrast gain control in the cat's visual system. J. Neurophysiol. 54, 651-667.

Pantle A. and Sekuler R. (1968) Size-detecting mechanisms in human vision. Science, N.Y. 162, 1146-1148.

Sclar G. and Lennie P. (1987) Contrast adaptation in Macaque visual cortex. Imvest, ophthal vistual Sci. (Suppl.), 28, 198.

Sclar G., Ohzawa I. and Freeman R. D. (1985) Contrast gain control in the kitten's visual system. $J$. Neurophysiol. 54, 668-675. 